Compte rendu de The Aranda's Pepa. An Introduction to Carl Strehlow's masterpiece 'Die Aranda- LoritjaStämme in Zentral-Australien', de Anna KENNY Laurent Dousset

\title{
OpenEdition
}

1 Journals

Édition électronique

URL : http://journals.openedition.org/jso/7398

DOI : $10.4000 /$ jso.7398

ISSN : $1760-7256$

Éditeur

Société des océanistes

Édition imprimée

Date de publication : 15 décembre 2015

Pagination : 351-353

ISBN : 978-2-85430-126-7

ISSN : 0300-953x

Référence électronique

Laurent Dousset, «Compte rendu de The Aranda's Pepa. An Introduction to Carl Strehlow's masterpiece 'Die Aranda- Loritja-Stämme in Zentral-Australien', de Anna kENny ", Journal de la Société des Océanistes [En ligne], 141 | juillet-décembre 2015, mis en ligne le 03 janvier 2016, consulté le 24 septembre 2020 URL : http://journals.openedition.org/jso/7398 ; DOI : https://doi.org/10.4000/jso.7398

(c) Tous droits réservés 
immédiatement après à Tahiti ne lui suffirent évidemment à maîtriser ce pidgin, et il est très probable qu'il en apprit les rudiments soit dans la navigation précédant son débarquement « sauvage ", soit dans les suivantes qui le mirent en contact avec le pidgin hawaiien dont quelques traces sont perceptibles dans ses notations. Gage supplémentaire d'authenticité, les " $\mathrm{r}$ " dont il truffe ses citations polynésiennes montrent qu'il ne les a pas puisées dans la littérature antérieure, et que s'il avait si " mauvaise oreille ", la cause en remontait aux particularités du parler entendu dans son enfance, à New York et quelques autres lieux de Nouvelle-Angleterre.

Dans la dernière partie (Structure, function, and history of Maritime Polynesian Pidgin), E. Drechsel revient successivement sur les caractéristiques syntaxiques et lexicales de ce pidgin (chapitre 7 , Linguistic patterns), sur ses divers rôles et usages (chapitre 8, History and social functions) et enfin sur les pistes ouvertes par cette enquête (chapitre 9, Conclusions: linguistic, sociohistorical, and theoretical implications). Sur le plan de la syntaxe, l'ordre sujet-verbe-objet (ou sujet-groupe verbal, sujet-copule-prédicat, etc.) n'est pas un critère décisif d'origine occidentale, cette structure coexistant en polynésien avec l'ordre verbe-sujetobjet, ou verbe-sujet, plus fréquent. Encore fautil spécifier dans quel contexte social, dans quel type de discours ou niveau de langue ils se trouvaient respectivement employés, et E. Drechsel a largement raison de souligner tout ce qui séparait le commerce avec les étrangers en lingua franca d'usages de la parole plus solennels ou touchant même au sacré, et dont les visiteurs occidentaux n'ont quasiment rien su recueillir. Du point de vue lexicographique, les conclusions de ce travail s'appuient sur un double répertoire alphabétique en même temps qu'analytique établi et mis en ligne par l'auteur, aujourd'hui consultable dans une version révisée (Drechsel, 2014).

Du point de vue historique, on s'explique mieux que ce pidgin, souvent cité par les Occidentaux pour apporter une pointe d'authenticité et de couleur locale à leurs récits des mers du Sud, ait jusqu’à présent échappé aux regards de beaucoup de spécialistes, du fait du statut spécial de ses locuteurs et de la relative brièveté de son existence :

"Based on currently available historical information, MPP did not carry on in New Zealand beyond the first half of the nineteenth century, and faced an uncertain fate in the Marquesas, even though declarations of demise remain premature without better evidence. The Pidgin still endured in the Hawaiian Islands on multiethnic plantations until the end of the nineteenth century and even into the early twentieth. » (p. 231)

Mais la conclusion probablement la plus importante de ce maître-livre d'ethnohistoire linguistique est que ce pidgin fut créé et déve- loppé à l'initiative même des insulaires, gardant ainsi quelque prise sur leurs visiteurs, tant qu'ils n'étaient que leurs hôtes, et non encore leurs envahisseurs, colonisateurs et évangélisateurs. Ainsi que le suggère le passage précité, sa disparition paraît coïncider remarquablement avec les prises de possession par les Européens des îles Marquises et de la Nouvelle-Zélande, derniers bastions de la résistance polynésienne à l'occidentalisation, et E. Drechsel semble avoir défini là un jalon essentiel, jusqu'à présent trop peu pris en compte, de l'histoire du Pacifique et de ses insulaires.

\section{RÉFÉRENCES CITÉES}

Claret de Fleurieu Charles Pierre, 1798. Voyage autour du monde pendant les années 1790, 1791, 1792, par Étienne Marchand..., Paris, imprimerie de la République.

Choris Louis, 1822. Voyage pittoresque autour du monde, avec des portraits de sauvages d'Amérique, d'Asie, d'Afrique, et des îles du Grand Océan..., Paris, Firmin Didot.

DreChSel Emanuel J. 2014. Etymological Vocabulary and Index of Maritime Polynesian Pidgin, Cambridge, Cambridge University Press (www. cambridge.org/ru/download_file/811732/).

INGRAHAM Joseph, 1971. Journal of the Brigantine Hope on a Voyage to the Northwest Coast of North America 1790-92, Barre, Massachusetts, Imprint Society.

Lutké Frédéric, 1835. Voyage autour du monde exécuté sur l'ordre de sa majesté l'empereur Nicolas Ir sur la corvette Le Séniavine..., tome second, Paris, Firmin Didot.

Gilles Bounoure

Kenny Anna, 2013. The Aranda's Pepa. An Introduction to Carl Strehlow's masterpiece 'Die Aranda-Loritja-Stämme in Zentral-Australien', Canberra, ANU E Press, XIX + 310 p., bibliogr., index.

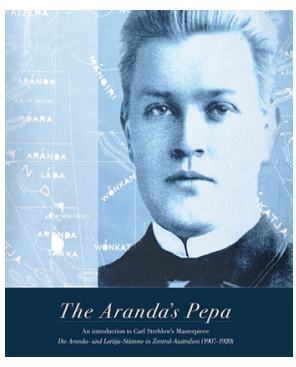

Sans aucun doute l'ethnographie des Aborigènes d'Australie, et plus particulièrement celle qui concerne la ou plutôt les tribus Aranda (Arrernte), a-t-elle marqué l'évolution des sciences humaines et sociales et influencé des personnages, concepts et théories fondateurs pour ces disciplines. On rappellera les formes élémentaires de la vie religieuse (Durkheim, 1912), les structures élémentaires de la parenté (Lévi-Strauss, 1947), ou encore Malthus, Morgan, Freud et bien d'autres encore qui tous, à un moment ou un autre et à des degrés 
divers ont pensé devoir, comme le disait si bien Aram Yengoyan (1979), confronter leurs hypothèses au terrain australien pour leur fournir la validité générale qu'ils espéraient.

Dans cette généalogie d'un assortiment important de la discipline anthropologique, les travaux de Spencer et Gillen sur les Aranda australiens apparaissent comme une source originelle et inévitable, affirmant raconter ce que ces Aborigènes étaient avant... avant l'arrivée des Blancs, de la colonisation, la déculturation, la sédentarisation, etc. Tout anthropologue comparatiste ou théoricien se doit, à un moment ou une autre, croiser leurs chemins ; celui des Aranda et de Spencer et Gillen, témoins inconditionnels d'un monde qui n'existerait plus mais qui, au-delà de la seule Australie, nous renverrait vers l'essentiel et le fondamental de l'humanité tout entière.

Derrière ce qu'on appellerait de nos jours la " médiatisation » inconditionnelle des travaux de ces deux auteurs, dont le premier, Walter B. Spencer, naturaliste et théoricien du darwinisme, doit tout au second, Francis J. Gillen, ethnographe assidu et méticuleux, se profile aussi une anthropologie particulière et particulièrement durable. Britannique de nationalité, systémique, presque structurale de par les modèles descriptifs et explicatifs déployés, cette science allait construire - pour ne pas dire inventer - des modèles dans lesquels, comme le traduisait Durkheim lorsqu'il affirmait la disparition de l'individu australien, les acteurs et interlocuteurs s'éteignent derrière des schémas, des catégories et des structures qui les dépassent à tel point que la stratégie, l'inflexion, l'adaptation, le changement ou, pire, la transformation seraient hors de portée de la conscience humaine (au moins aborigène).

L'anthropologie, et plus particulièrement australianiste, aurait probablement été tout autre si l'histoire avait voué autant de passion et d'importance à un autre personnage, de la même époque et travaillant avec la même tribu, à savoir le missionnaire luthérien Carl Strehlow (18711922). Restée non traduite en anglais et dénigrée avec virulence par Spencer qui avait, et ceci de manière explicite, décidé d'entrer dans l'histoire comme le dernier qui avait vu et vécu les véritables Aranda, l'œuvre gigantesque de Strehlow, comme de tant de missionnaires luthériens, si importante et fondamentale pourtant, fut tout simplement oubliée... ou presque.

Complémentaire parfois, souvent contraire aux généralisations d'un Spencer, l'ethnographie de Strehlow est tout le contraire de ces darwinismes et systématismes que prône à l'époque l'approche britannique. Humaniste de formation, Strehlow s'intéresse aussi à tous ces aspects de la culture Aranda, oubliés ou évacués par Spencer et Gillen, qui mettent au cour de la réflexion les nuances linguistiques, les fluidités sociales et les actions et pensées des individus à tel point qu'on peut par- ler ici d'une tentative précoce de dégager une véritable ontologie des représentations aborigènes. Strehlow était un des émissaires de ce même humanisme qui plaçait la langue au cœur de l'intelligibilité culturelle auquel appartenait Boas.

Issu d'une thèse de doctorat, c'est à la découverte ou redécouverte de cette œuvre que l'ouvrage d'Anna Kenny est voué. Ayant travaillé sur et avec les Aranda, en particulier dans le cadre de revendications foncières, l'auteur découvre les archives et surtout l'importante correspondance entre le missionnaire Carl Strehlow et le scientifique Moritz von Leonhardi - éditeur, mentor et ami du premier - qui sont hébergés au Strehlow Research Center à Alice Springs, en Australie centrale. Cette découverte et la lecture de Die Aranda-Loritja-Stämme in Zentral-Australien, publié de 1907 à 1920 en cinq volumes, permettent à Kenny de redonner à Strehlow toute sa place dans l'ethnographie australienne et au-delà.

Louvrage est organisé en deux parties avec chacun plusieurs chapitres. La première partie nous raconte la vie de Carl Strehlow, ses séjours en Australie, en particulier à la mission de Hermannsburg, et la manière dont sa pensée et sa méthode ethnographique furent progressivement modelées au travers de sa formation religieuse et de ses échanges avec von Leonhardi. Anna Kenny replace ainsi les travaux de Strehlow dans l'histoire de traditions anthropologiques divergentes. Allemande d'une part, inspirée de Gottfried Herder en particulier, qui fonda une approche dans laquelle l'analyse des particularités culturelles, indissociables de la langue, devient à elle seule l'objectif premier du travail ethnographique et analytique. C'est cette tradition qui, au travers de Boas, motiva également un pan important de l'anthropologie outre-Atlantique. Tradition britannique de l'autre, où, au contraire, les particularités culturelles sont saisies comme des témoins ou des vestiges d'une chaine évolutive (et plus tard fonctionnaliste et structurelle) qui ne peut être comprise que par la juxtaposition de systèmes culturels comparés. Si la première tradition tente de détailler les manières de faire et de penser propres aux individus porteurs d'une culture, la seconde au contraire s'intéressera aux aspects structurels et systémiques qui peuvent engendrer des regards croisés. L'académie australienne étant dominée par cette seconde tradition, l'analyse de Kenny nous permet de mieux comprendre le contexte dans lequel une ouvre comme celle de Strehlow ne pouvait connaître autre destin que la négligence.

La seconde partie présente et analyse un éventail d'aspects ethnologiques et éléments ethnographiques des travaux de Strehlow en les replaçant dans une exigence anthropologique plus contemporaine. Tout en soulignant les limites de certaines propositions du missionnaire-chercheur, en particulier celles du domaine de la parenté pour laquelle Strehlow ne semblait pas avoir déve- 
loppé une sensibilité suffisante, elle montre aussi combien l'analyse des mythes, de la cosmologie, des rapports fonciers et des classifications sociales a tout à gagner à une lecture de ces travaux pourtant centenaires. Alors que, pour ne citer qu'un domaine, Spencer et Gillen - et plus tard Radcliffe-Brown dans la même lignée - décrivaient un système d'organisation territoriale figé dans des règles pensées objectives et déterminées, l'ethnographie de Strehlow montre au contraire à quel point les filiations complémentaires, les stratégies et les fluidités, ainsi que les affiliations véritables des individus sont les témoins de modalités de transmission et de reproduction d'organisations foncières complexes et souples à la fois. L'ethnographie de Strehlow fait comprendre, s'il en était encore besoin, que même ces Aborigènes qui n'avaient pas encore subi les transformations induites par l'arrivée de la société occidentale, étaient déjà de véritables acteurs qui savaient jongler avec les normes et les valeurs, loin de n'être que des pions de structures sociales inaltérables.

Malgré quelques imperfections et parfois des passages et transitions trop rapides, l'ouvrage d'Anna Kenny constitue ainsi et sans aucun doute une contribution importante pour l'anthropologie australianiste et au-delà. Il nous fournit la clé pour découvrir et comprendre toute une ethnographie oubliée et pourtant de grande importance. À la lecture de cet ouvrage, on ne peut que regretter qu'il n'y ait pas eu davantage de Carl Strehlow ailleurs également.

\section{RÉFÉRENCES CITÉES}

Durкheim Émile, 1960 (1912). Les formes élémentaires de la vie religieuse. Le système totémique en Australie, Paris, PUf.

LÉvi-Strauss Claude, 1947. Les structures élémentaires de la parenté, Paris, Mouton.

Yengoyan Aram, 1979. Economy, Society, and Myth in Aboriginal Australia, Annual Review of Anthropology 8, pp. 393-415.

Laurent DousseT, Marseille, CREDO EHESS

Parke Aubrey, 2014. Degei's Descendants. Spirits, Place and People in Pre-Cession Fiji, edited by Matthew Spriggs and Deryck Scarr, Canberra, ANU Press, xxx-308 p., cartes, appendices, bibliogr.

La présence des éditeurs sur la page de titre le suggère, il s'agit d'une publication posthume, préparée par leurs soins à partir de la thèse soutenue par Aubrey Parke (1925-2007) dans la dernière année de sa vie, où il continuait de lutter contre une maladie sans remède. Comme l'expose Matthiew Spriggs dans l'hommage biographique ouvrant le volume, ce titre universitaire couronnait plus d'un demi-siècle de recherches passionnées sur l'archipel fidjien, son histoire et sa préhistoire. Après de solides études classiques, qui lui firent mener des fouilles archéologiques dans le Dorset, et des années de guerre où il mit sa vie en jeu à bord de bombardiers de la RAF, Parke prolongea ses études à Oxford et fut recruté par le British Colonial Service qui l'envoya aux îles Fidji. Il y occupa divers postes dans les deux décennies précédant l'indépendance (octobre 1970), sans cesser de se soucier d'archéologie, devenant même le conseiller du Fiji Museum en la matière. Il s'établit ensuite à Canberra, intégra l'administration de la future université nationale, continua à se préoccuper de la préhistoire fidjienne, mais ne put se consacrer entièrement à l'œuvre qu'il projetait qu'en 1990, au moment de sa retraite, avant que sa maladie n'interfère dans l'avancement de son travail. C'est à l'hôpital que lui furent remis ses insignes de docteur.

Les contributions de Parke à l'archéologie fidjienne sont restées peu ou prou ignorées et manquent à la plupart des bibliographies récentes. M. Spriggs confesse lui-même ne pas en avoir pris connaissance avant de se charger d'éditer ce volume. Dans un panorama pourtant extrêmement détaillé des débuts de l'archéologie en Polynésie occidentale, Janet Davidson (2008 : 23) écrit seulement de lui :

"Long time resident Aubrey Parke reported on ceremonial sites on Vanua Levu (Parke 1971, 1972). "

sans mention de ses publications ultérieures. En tête d'un volume collectif spécifiquement dévolue à l'archéologie fidjienne, Geoffrey Clark et Atholl Anderson l'incluent dans leurs remerciements (2009: 13) mais ne citent aucun de ses travaux, etc. Cet état de fait a conduit M. Spriggs à vérifier si Parke était seulement "an enthusiastic amateur in Fiji ", comme l'écrivait Simon Best à propos de ses études des fortifications fidjiennes (JPS 102-4, 1993 : 396). Et de montrer que loin d'être un amateur, il était probablement, de tous les "résidents " britanniques de l'archipel dans les décennies précédant l'indépendance, le mieux formé aux recherches archéologiques, et l'un des plus aptes à recueillir des insulaires leurs traditions ethno-historiques, comme il sut le faire à Rotuma. Mais il était fonctionnaire de l'administration coloniale et non chercheur attitré ou envoyé en mission, et son scepticisme à l'égard des

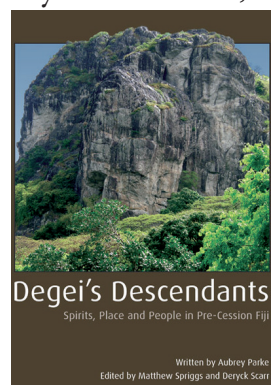
datations par radiocarbone et autres méthodes de laboratoire mises en avant par les archéologues de l'après-guerre soucieux de promouvoir leur discipline le plaçait forcément en marge de la recherche académique et de ses réseaux.

Pour être touchante, sinon exemplaire de la part de son 